\title{
Mechanical control of the mammalian circadian clock via YAP/TAZ and TEAD
}

Juan F. Abenza ${ }^{1,2, \ddagger *}$, Leone Rossetti ${ }^{1,}$, Malèke Mouelhi ${ }^{1}$, Javier Burgués ${ }^{1}$, Ion Andreu ${ }^{1}$, Keith Kennedy ${ }^{3}$, Pere Roca-Cusachs ${ }^{1,4}$, Santiago Marco ${ }^{1,5}$, Jordi García-Ojalvo ${ }^{3}$ and Xavier Trepat $^{1,2,4,6, *}$

${ }^{1}$ Institute for Bioengineering of Catalonia (IBEC), The Barcelona Institute for Science and Technology (BIST), 08028 Barcelona, Spain.

${ }^{2}$ Centro de Investigación Biomédica en Red en Bioingeniería, Biomateriales y Nanomedicina (CIBER-BBN), 08028 Barcelona, Spain.

${ }^{3}$ Department of Experimental and Health Sciences, Universitat Pompeu Fabra, 08003 Barcelona, Spain.

${ }^{4}$ Facultat de Medicina, Universitat de Barcelona, 08036 Barcelona, Spain.

${ }^{5}$ Department of Electronics and Biomedical Engineering, Universitat de Barcelona, 08028 Barcelona, Spain.

${ }^{6}$ Institució Catalana de Recerca i Estudis Avançats (ICREA), Barcelona, Spain.

$\$$ These authors contributed equally to this work

* Correspondence to:

Xavier Trepat, $\mathrm{PhD}$

Institute for Bioengineering of Catalonia

Ed. Hèlix, Baldiri i Reixac, 15-21

Email: xtrepat@ibecbarcelona.eu

Juan Francisco Abenza, PhD

Institute for Bioengineering of Catalonia

Ed. Hèlix, Baldiri i Reixac, 15-21

Email: jabenza@ibecbarcelona.eu 


\begin{abstract}
Circadian rhythms are a key survival mechanism that dictates biological activity according to the day-night cycle ${ }^{1}$. In animals, cell-autonomous circadian clocks can be found in nearly every cell type and are subjected to multi-layered regulation ${ }^{2-4}$. Although these peripheral clocks are remotely controlled by the master clock in the brain, they are also sensitive to their immediate physical microenvironment through mechanisms that are still unknown ${ }^{5,6}$. Here we show that the circadian clock in fibroblasts is regulated mechanically through YAP/TAZ and TEAD. We use high-throughput analysis of single-cell circadian rhythms and apply controlled mechanical, biochemical, and genetic perturbations to study the expression of the core clock gene Rev$e r b \alpha^{7}$. We observe that Rev-erb $\alpha$ circadian oscillations are disrupted concomitantly with the translocation of YAP/TAZ to the nucleus. By targeted mutations and tuning expression levels of YAP we identify TEAD as the transcriptional effector of this mechanosensitive regulatory pathway. Our findings establish a mechanism that links cell mechanobiology and the circadian clock, which could contribute to explain the circadian impairment observed in cancer and ageing, where the regulation of the mechanical environment and YAP/TAZ is $\operatorname{lost}^{8-11}$.
\end{abstract}

\title{
Main text
}

Circadian rhythms are defined as an organism's physiological and behavioural adaptations to the cyclic environment that arises from the Earth's rotation ${ }^{1}$. In mammals, these rhythms are intrinsically sustained by the circadian clock, a set of proteins whose expression is selfregulated, at the single-cell level, via a transcription-translation feedback loop with a period close to 24 hours $^{12}$. Up to $25 \%$ of the mammalian proteome is estimated to be influenced, in a cell type-specific manner, by the circadian clock, which deeply conditions dynamics and function of tissues and organs throughout the day ${ }^{13-15}$.

The central element of the mammalian circadian clock is the transcription factor BMAL1:CLOCK, which activates the transcription of, among many others, Cry and Per. In turn, the sustained expression of CRY and PER generates a transcriptional loop by binding to BMAL1:CLOCK and blocking its activity ${ }^{16}$. A second stabilizing layer of regulation of the clock is provided by REV-ERB, which, on one hand, is transcriptionally promoted by BMAL1:CLOCK and, on the other hand, acts as a transcriptional inhibitor of Bmall $^{7}$.

For every cell residing in a peripheral tissue, the robustness of the circadian clock and its synchrony with the environment are subjected to multi-layered regulation ${ }^{2,3}$. Decades of work have unveiled the biochemical side of this regulatory network which, mainly through neurocrine and paracrine signalling, drives important changes in the gene expression landscape of the different cell types ${ }^{4,17,18}$. Besides biochemical regulation, recent evidence suggests that the circadian clock is also influenced by mechanical factors such as actin dynamics, cell-cell adhesions and the stiffness of the extracellular matrix $(\mathrm{ECM})^{5,6,19}$. However, the mechanosensitive molecular mechanisms that enable the transcriptional regulation of the circadian network are unknown.

By combining confocal microscopy, microfabrication, and a customized computational toolkit, we show that high nuclear levels of the transcriptional regulators YAP/TAZ cause a dramatic 
change in the expression of Rev-erb $\alpha$ and the subsequent malfunction of the fibroblast circadian clock. This novel molecular mechanism, which we show to be TEAD-dependent, could explain the highly impaired circadian clock in circumstances typically associated with high nuclear YAP/TAZ translocation such as cancer or ageing ${ }^{8-11}$.

We conducted our work using NIH3T3 fibroblasts, a cellular model extensively reported to express a robust self-sustained circadian molecular clock $^{20,21}$. To prevent the dominance of specific signalling pathways derived from hormonal shocks ${ }^{22}$ and reveal underlying mechanobiological signals, cells were not synchronized by exogenous entrainment during the experiment. We generated a stable clonal cell line carrying both a reporter of Rev-erb $\alpha$ transcription (RevVNP) ${ }^{20}$ and a chimeric histone2B-mCherry that we used as a constitutive nuclear marker (Fig. 1a, left). We also developed a method to systematically track and measure the single cell expression of RevVNP over 3-day time-lapses performed by confocal microscopy. Then, by performing Fourier analysis of the fluorescence emission of each cell and quantifying the fraction of the power-spectrum belonging to the circadian frequency, we obtained a signature of the circadian strength of every single cell (Fig. 1a, Methods).

We first addressed the effect of cell density on RevVNP expression by culturing cells on hydrogels (30 $\mathrm{kPa}$ in stiffness) at two distinct densities $\left(\sim 30\right.$ cells $/ \mathrm{mm}^{2}$ and 1000 cells $/ \mathrm{mm}^{2}$, hereafter referred to as low and high density, respectively), which we kept approximately constant over time by arresting the cell cycle with the addition of thymidine, a DNA synthesis inhibitor $^{23}$. We observed a dramatic cell density-dependent difference in Rev-erb $\alpha$ expression, with cells cultured at low density displaying much higher RevVNP nuclear intensity than confluent cells (Fig. 1b-c). These differences were ratified at the protein level via immunostainings against REV-ERB $\alpha$ (Extended Data Fig. 1). The single cell frequency analysis of the RevVNP signal revealed that the population of cells grown at high density expressed robust oscillations with an average period of $24.7 \pm 1.9 \mathrm{~h}$ (Fig. 1d-e). By contrast, cells at low density displayed low RevVNP circadian strength, with many cells exhibiting poor circadian oscillations, missing one or several peaks or even experiencing non-periodic fluctuations rather than oscillatory behaviour (Fig. 1d-e).

We next asked whether the detected differences in circadian strength are caused by paracrine signals, as previously reported for intercellular circadian coupling and synchronization ${ }^{24,25}$. To address this question, we exposed closely packed cells to an abrupt drop in cell density. To do so, two cell monolayers were grown to confluence separated by a PDMS barrier. Upon lifting the barrier (Fig. 2a, left), cells at the monolayer edges begun to spread and migrate towards the freely available gap. These cells experienced a sudden increase in RevVNP intensity that transiently disrupted their circadian oscillations until the gap was closed (Fig. 2a-c; Supplementary Video 1). This disruption contrasted with the behaviour of cells far from the edge, which oscillated robustly throughout the entire experiment as shown in the kymograph depicted in Fig. 2b. We obtained similar results in presence or absence of thymidine (Extended Data Fig. 2), which confirms that the increase seen in Rev-erb $\alpha$ transcription is not a side effect of a cell cycle re-entry at the monolayer edge upon a loss of cell-cell contact. These experiments further demonstrate that cell density regulates the circadian clock and suggest that this phenomenon is not explained by paracrine signals, as high- and low-density cells shared the 
same extracellular chemical environment. We confirmed that changes in paracrine signalling are not the cause of RevVNP circadian impairment by growing cells at low density with conditioned medium obtained from a high-density culture. We observed no increase in circadian strength compared to the low-density cells grown in fresh medium (Fig. 2d). These results indicate that cell density does not affect the circadian expression of Rev-erb $\alpha$ through paracrine signals, which led us to hypothesize that the observed behaviour had a mechanochemical origin.

To test this hypothesis, we first asked whether the differences in RevVNP circadian oscillations between high and low density depend on cell-cell adhesion. To address this question, we confined individual cells in fibronectin micropatterns of $1200 \mu \mathrm{m}^{2}$ (a size comparable to that of fibroblasts within a monolayer) (Fig. 2e). The confined individual cells showed a significant reduction in RevVNP intensity together with an increase in circadian strength when compared to free cells grown at equivalent low density, a behaviour resembling that of high-density cells (Fig. 2e-f; Supplementary Video 2). This result implies that contact-based mechanisms in general, and intercellular adhesion in particular, do not explain the dependence of the Rev-erba circadian oscillations on cell density.

We thus explored whether the onset of circadian oscillations at high density had a contactindependent mechanical origin. We focussed on two key mechanosensitive transcriptional regulators. The first one is MAL, whose localization is regulated by actin dynamics and has been shown to modulate the transcription of the circadian gene $P e r 2^{26}$. The second one is YAP, the central component of the Hippo pathway, which translocates in a mechanoresponsive manner between the cytosol and the nucleus ${ }^{27-29}$. YAP was recently shown to bind to REV$\mathrm{ERB} \alpha^{30}$ although it has not been linked to the circadian clock. We measured and compared both circadian strength and subcellular localization of MAL and YAP in a series of conditions known to impact their nucleocytoplasmic shuttling. These conditions include the already mentioned high and low cell density and single cell micropatterning; treatment with drugs that alter the actomyosin cytoskeleton such as jasplakinolide, latrunculin A, para-nitro-blebbistatin and cytochalasin $\mathrm{D}$; and the growth of cells on very soft hydrogels $(300 \mathrm{~Pa})^{28,31-33}$. As expected, these conditions had a variety of effects on cell morphology (Fig. 3a, Extended Data Fig. 3a). We found no correlation between nuclear to cytoplasmic ratio of MAL and circadian strength (Extended Data Fig. 3). By contrast, circadian strength showed a progressive decrease with YAP nuclear to cytoplasmic ratio (Fig. 3). This striking anticorrelation reveals that the robustness of the Rev-erb $\alpha$ circadian expression depends on the nucleocytoplasmic transport of YAP and its mechanosensitive regulation.

To test whether this dependence is causal, we induced in high-density cells a sustained overexpression of a non-phosphorylatable YAP mutant, 5SA-YAP, which has abnormally high nuclear retention ${ }^{34}$. The resulting high levels of 5SA-YAP provoked a severe reduction of RevVNP circadian strength (Fig. 4a) and an increase in REV-ERB $\alpha$ basal levels when compared to high-density control cells (Fig. 4b-d). A similar result was obtained when overexpressing the TAZ mutant 4SA-TAZ, which is also retained in the nucleus due to the mutation of its four Lats inhibitory phosphorylation sites ${ }^{35,36}$ (Extended Data Fig. 4). Together, these results establish that the circadian clock is controlled through both YAP and TAZ. 
The activity of YAP/TAZ as gene expression coactivators requires the interaction with other transcription factors. Since YAP interacts with REV-ERB $\alpha$ through its coiled-coil (CC) domain $^{30}$, we tested if this interaction gives rise to the deleterious effect of nuclear YAP on the circadian clock. For that, we overexpressed 5SA-YAP but with its CC domain deleted (5SACCDEL-YAP), a mutation that had been previously shown to disable the molecular interaction between YAP and REV-ERB $\alpha^{30}$. We found that the CCDEL mutation only rescued slightly the impaired RevVNP circadian oscillations (Fig. 4a) and the REV-ERB $\alpha$ basal levels (Fig. 4b-d) observed upon 5SA-YAP overexpression. This result shows that the direct molecular binding through the YAP CC domain is not the main regulatory pathway connecting YAP and the circadian clock.

Alternatively, we studied whether this regulation was mediated by the well-known interaction of YAP/TAZ with the TEAD family ${ }^{37}$. Analysis of published ChIP-seq and microarray databases revealed that Bmall -but not Rev-erb $\alpha$ - is listed as a YAP and TEAD target ${ }^{38-41}$, providing a potential direct mechanism for YAP/TAZ to control the circadian clock. To test this possibility, we overexpressed 5SA-S94A-YAP, a mutant version of YAP unable to interact with $\mathrm{TEAD}^{40}$ and observed that the cells recovered, to a large extent, both the RevVNP circadian strength and the REV-ERB $\alpha$ basal levels displayed by the wild-type high-density population (Fig. 4). This result establishes that the circadian clock deregulation in nuclear YAP-enriched cells is caused by the YAP-TEAD transcriptional cascade.

Our discovery that nuclear YAP/TAZ disrupts Rev-erb $\alpha$ oscillations provides an entirely new layer of regulation for the circadian clock. Given the widespread role of YAP/TAZ in physiology and disease, the potential implications of this new mechanism are multiple. For example, this mechanism could contribute to explain the loss of circadian rhythms in ageing and cancer, two conditions in which YAP/TAZ become activated ${ }^{42-45}$. Moreover, given the role of REV-ERB $\alpha$ in endocrine function, fat storage, thermogenesis, mitochondrial activity, and cholesterol $^{46}$, we expect this study to contribute new ways to understand the intimate relationship between circadian clocks and metabolism. Considering the role of YAP/TAZ as core mechanosensors, our findings provide a fundamental link between the largely disconnected fields of chronobiology and mechanobiology. 


\section{Main references}

1. Bass, J. \& Lazar, M. A. Circadian time signatures of fitness and disease. Science vol. 354 (2016).

2. $\quad$ Finger, A. M., Dibner, C. \& Kramer, A. Coupled network of the circadian clocks: a driving force of rhythmic physiology. FEBS Letters vol. 594 (2020).

3. Albrecht, U. Timing to Perfection: The Biology of Central and Peripheral Circadian Clocks. Neuron vol. 74 (2012).

4. Reinke, H. \& Asher, G. Crosstalk between metabolism and circadian clocks. Nature Reviews Molecular Cell Biology vol. 20 (2019).

5. Streuli, C. H. \& Meng, Q. J. Influence of the extracellular matrix on cell-intrinsic circadian clocks. Journal of Cell Science vol. 132 (2019).

6. Hoyle, N. P. et al. Circadian actin dynamics drive rhythmic fibroblast mobilization during wound healing. Sci. Transl. Med. 9, (2017).

7. Preitner, N. et al. The orphan nuclear receptor REV-ERB $\alpha$ controls circadian transcription within the positive limb of the mammalian circadian oscillator. Cell 110, 251-260 (2002).

8. Stearns-Reider, K. M. et al. Aging of the skeletal muscle extracellular matrix drives a stem cell fibrogenic conversion. Aging Cell 16, (2017).

9. Zanconato, F., Cordenonsi, M. \& Piccolo, S. YAP/TAZ at the Roots of Cancer. Cancer Cell vol. 29 (2016).

10. Mattis, J. \& Sehgal, A. Circadian Rhythms, Sleep, and Disorders of Aging. Trends in Endocrinology and Metabolism vol. 27 (2016).

11. Blakeman, V., Williams, J. L., Meng, Q. J. \& Streuli, C. H. Circadian clocks and breast cancer. Breast Cancer Research vol. 18 (2016).

12. Cox, K. H. \& Takahashi, J. S. Circadian clock genes and the transcriptional architecture of the clock mechanism. J. Mol. Endocrinol. 63, (2019).

13. Storch, K.-F. et al. Extensive and divergent circadian gene expression in liver and heart. Nature 417, 78-83 (2002).

14. Rijo-Ferreira, F. \& Takahashi, J. S. Genomics of circadian rhythms in health and disease. Genome Medicine vol. 11 (2019).

15. Beytebiere, J. R. et al. Tissue-specific BMAL1 cistromes reveal that rhythmic transcription is associated with rhythmic enhancer-enhancer interactions. Genes Dev. 33, (2019).

16. Shearman, L. P. et al. Interacting molecular loops in the mammalian circadian clock. Science (80-. ). 288, (2000). 
17. Dibner, C., Schibler, U. \& Albrecht, U. The mammalian circadian timing system: Organization and coordination of central and peripheral clocks. Annual Review of Physiology vol. 72 (2009).

18. Koronowski, K. B. et al. Defining the Independence of the Liver Circadian Clock. Cell 177, (2019).

19. Yang, N. et al. Cellular mechano-environment regulates the mammary circadian clock. Nat. Commun. 8, 1-13 (2017).

20. Nagoshi, E. et al. Circadian gene expression in individual fibroblasts: Cellautonomous and self-sustained oscillators pass time to daughter cells. Cell 119, 693705 (2004).

21. Bieler, J. et al. Robust synchronization of coupled circadian and cell cycle oscillators in single mammalian cells. Mol. Syst. Biol. 10, 739 (2014).

22. Balsalobre, a, Damiola, F. \& Schibler, U. A serum shock induces circadian gene expression in mammalian tissue culture cells [see comments]. Cell 93, 929-937 (1998).

23. Jackman, J. \& O'Connor, P. M. Methods for Synchronizing Cells at Specific Stages of the Cell Cycle. Curr. Protoc. Cell Biol. (1998) doi:10.1002/0471143030.cb0803s00.

24. Noguchi, T., Wang, L. L. \& Welsh, D. K. Fibroblast PER2 circadian rhythmicity depends on cell density. J. Biol. Rhythms 28, 183-192 (2013).

25. Finger, A. M. et al. Intercellular coupling between peripheral circadian oscillators by TGF- $\beta$ signaling. Sci. Adv. 7, (2021).

26. Gerber, A. et al. Blood-borne circadian signal stimulates daily oscillations in actin dynamics and SRF activity. Cell 152, 492-503 (2013).

27. Aragona, M. et al. A mechanical checkpoint controls multicellular growth through YAP/TAZ regulation by actin-processing factors. Cell 154, 1047-1059 (2013).

28. Elosegui-Artola, A. et al. Force Triggers YAP Nuclear Entry by Regulating Transport across Nuclear Pores. Cell 171, 1397-1410.e14 (2017).

29. Dupont, S. et al. Role of YAP/TAZ in mechanotransduction. Nature 474, (2011).

30. Zhang, X. et al. TFCP2 Is Required for YAP-Dependent Transcription to Stimulate Liver Malignancy. Cell Rep. 21, 1227-1239 (2017).

31. Wada, K. I., Itoga, K., Okano, T., Yonemura, S. \& Sasaki, H. Hippo pathway regulation by cell morphology and stress fibers. Development 138, 3907-3914 (2011).

32. Gegenfurtner, F. A. et al. Micropatterning as a tool to identify regulatory triggers and kinetics of actin-mediated endothelial mechanosensing. J. Cell Sci. 131, jcs212886 (2018). 
33. Sun, B. et al. Actin polymerization state regulates osteogenic differentiation in human adipose-derived stem cells. Cell. Mol. Biol. Lett. 26, (2021).

34. Zhao, B. et al. Inactivation of YAP oncoprotein by the Hippo pathway is involved in cell contact inhibition and tissue growth control. Genes Dev. 21, 2747-2761 (2007).

35. Park, H. W. et al. Alternative Wnt Signaling Activates YAP/TAZ. Cell 162, (2015).

36. Lei, Q.-Y. et al. TAZ Promotes Cell Proliferation and Epithelial-Mesenchymal Transition and Is Inhibited by the Hippo Pathway. Mol. Cell. Biol. 28, (2008).

37. Vassilev, A., Kaneko, K. J., Shu, H., Zhao, Y. \& DePamphilis, M. L. TEAD/TEF transcription factors utilize the activation domain of YAP65, a Src/Yes-associated protein localized in the cytoplasm. Genes Dev. 15, (2001).

38. Lee, D. H. et al. LATS-YAP/TAZ controls lineage specification by regulating TGF $\beta$ signaling and Hnf4 $\alpha$ expression during liver development. Nat. Commun. 7, (2016).

39. Rajbhandari, P. et al. Cross-cohort analysis identifies a TEAD4-MYCN positive feedback loop as the core regulatory element of high-risk neuroblastoma. Cancer Discov. 8, (2018).

40. Zhao, B. et al. TEAD mediates YAP-dependent gene induction and growth control. Genes Dev. 22, (2008).

41. Zanconato, F. et al. Genome-wide association between YAP/TAZ/TEAD and AP-1 at enhancers drives oncogenic growth. Nat. Cell Biol. 17, (2015).

42. Kondratov, R. V., Kondratova, A. A., Gorbacheva, V. Y., Vykhovanets, O. V. \& Antoch, M. P. Early aging and age-related pathologies in mice deficient in BMAL1, the core component of the circadian clock. Genes Dev. 20, (2006).

43. Salzer, M. C. et al. Identity Noise and Adipogenic Traits Characterize Dermal Fibroblast Aging. Cell 175, (2018).

44. Broadberry, E. et al. Disrupted circadian clocks and altered tissue mechanics in primary human breast tumours. Breast Cancer Res. 20, 1-13 (2018).

45. Rivera-Reyes, A. et al. YAP1 enhances NF-кB-dependent and independent effects on clock-mediated unfolded protein responses and autophagy in sarcoma. Cell Death Dis. 9, (2018).

46. Everett, L. J. \& Lazar, M. A. Nuclear receptor Rev-erba: Up, down, and all around. Trends in Endocrinology and Metabolism vol. 25 (2014). 


\section{Figures}

a
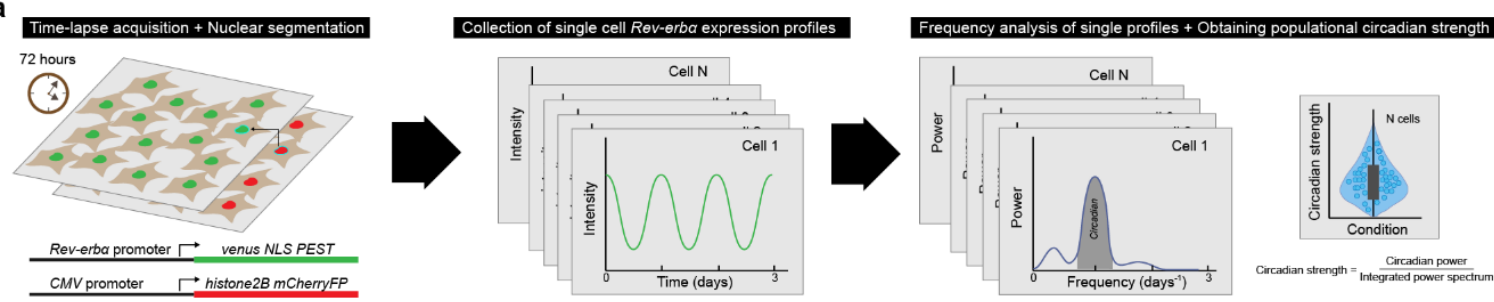

b

Low density

High density
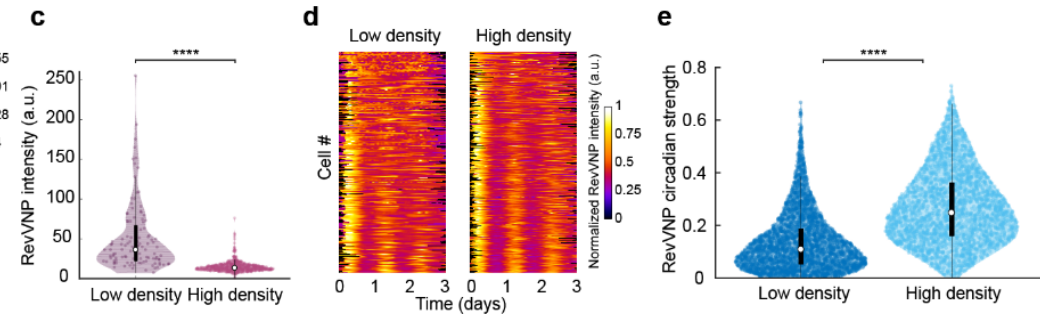

Fig. 1. Rev-erba basal expression and circadian oscillations depend on cell density. a, Schematics of the systematic computational analysis pipeline used to calculate the circadian strength of a population of cells expressing RevVNP and H2B-mCherry and imaged during 72 hours via time-lapse confocal microscopy. b, Confocal microscopy (top) and phase contrast (bottom) images of RevVNP-expressing cells grown at low (left) and high (right) density. Scale bar, $50 \mu \mathrm{m}$. c, Violin plots representing the distribution of the single-cell RevVNP intensities of low- and high-density populations of a prototypic experiment of $14 ; n=112$ cells and $n=622$ for low density and high density, respectively; Medians and interquartile ranges are depicted as white circles and black bars, respectively. Two-sided Wilcoxon rank sum test; **** indicates a p-value $<0.001$. Full p-values are reported in Extended Data Table 1. d, Kymograph-style compilation of the RevVNP intensity over time of 325 cells grown and tracked under low- or high-density conditions, from a representative experiment of 14. The single tracks are ordered from less (top) to more (bottom) circadian strength and aligned along the time axis according to maximum cross-correlation with the median track. e, Violin plots representing the distribution of the single-cell RevVNP circadian strength of low- and high-density populations; $\mathrm{n}=2726$ and 2389 cells, respectively, from four experiments; Medians and interquartile ranges are depicted as white circles and black bars, respectively. Two-sided Wilcoxon rank sum test; **** indicates a p-value $<0.001$. Full p-values are reported in Extended Data Table 1. 
a
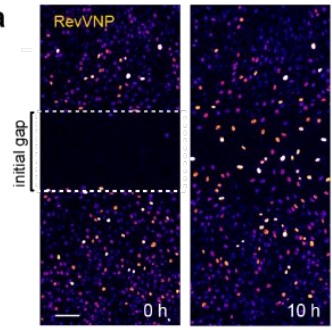

d

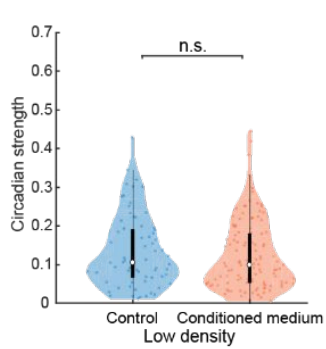

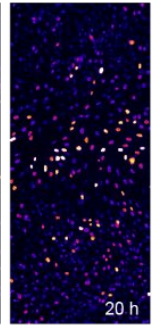
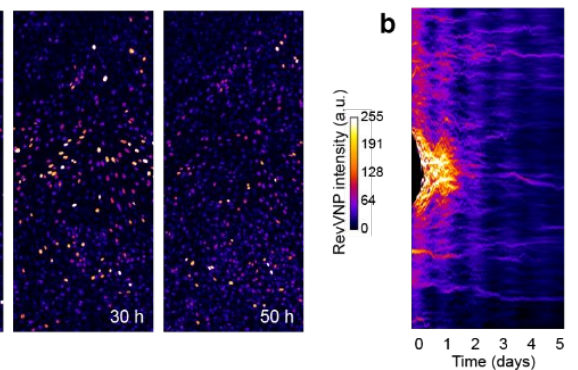

e

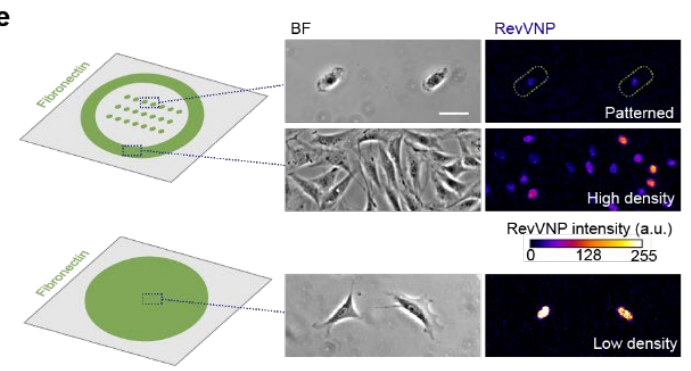

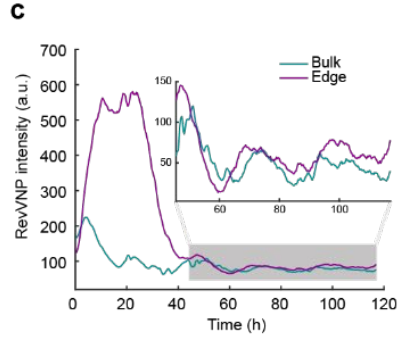

$\mathbf{f}$

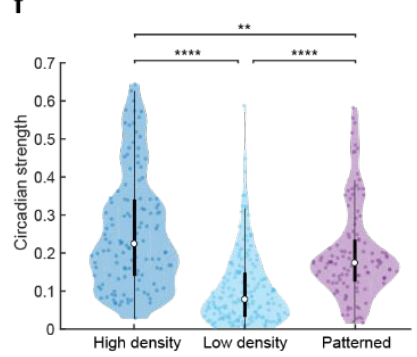

Fig. 2. Rev-erba circadian oscillations do not depend on cell-cell adhesions or paracrine signalling. a, RevVNP-expressing cells were cultured in two adjacent compartments separated by a barrier until they reached high density. Next, the barrier was removed, and time-lapse confocal microscopy was performed during and after gap closure every 15 minutes for 5 days. A sequence of time-lapse images corresponding to the closure of the gap is shown. The prior location of the barrier is depicted in the left image as a white dashed line. b, Kymograph representing the average RevVNP intensity per time point of all the cells from a along the $\mathrm{x}$ axis. c, Average RevVNP intensity over time of the cells at the edge in comparison to those residing in the confluent zone. The inset shows a magnification of the area shaded in grey. This figure shows an example of $\mathrm{n}=6$ independent experiments. Scale bar, $100 \mu \mathrm{m}$. d, Violin plots representing the distribution of the single-cell RevVNP circadian strengths of low-density cells grown in fresh medium and conditioned medium; $n=72$ and 116 cells, respectively, from three experiments; Medians and interquartile ranges are depicted as white circles and black bars, respectively; Two-sided Wilcoxon rank sum test; p-value $=0.4168$. e, Schematics of the strategy followed to isolate single cells via micropatterning of fibronectin on glass. On the right, phase contrast and confocal microscopy images of cells in stadium-shaped patterns (top), confluent cells cultured in the same well (middle), and non-confined cells cultured on a homogenous fibronectin-coated surface in a density as low as that of the micropatterned cells (bottom). Scale bar, $50 \mu \mathrm{m}$. f, Violin plots representing the distribution of the single-cell RevVNP circadian strengths of the conditions depicted in e); $\mathrm{n}=121,174$ and 115 cells for the high-density, low-density and micropatterned cells, respectively, from three experiments; Medians and interquartile ranges are depicted as white circles and black bars, respectively. Two-sided Wilcoxon rank sum test; ** indicates p-value $<0.01$; **** indicates p-values $<$ 0.0001. Full p-values are reported in Extended Data Table 1. 
a

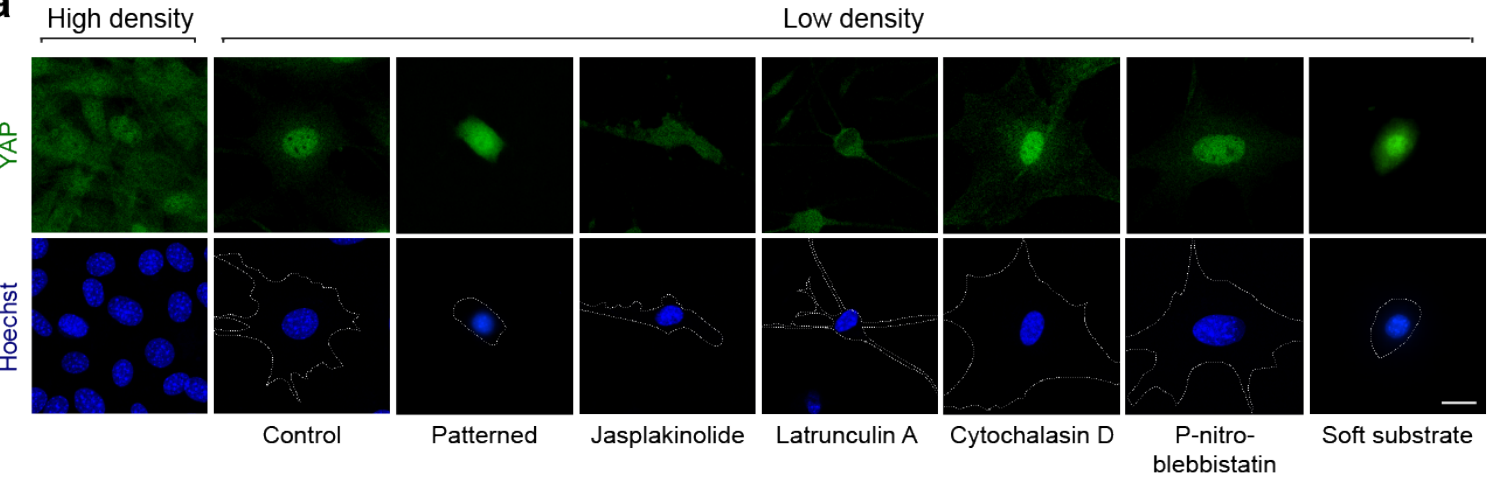

b

C
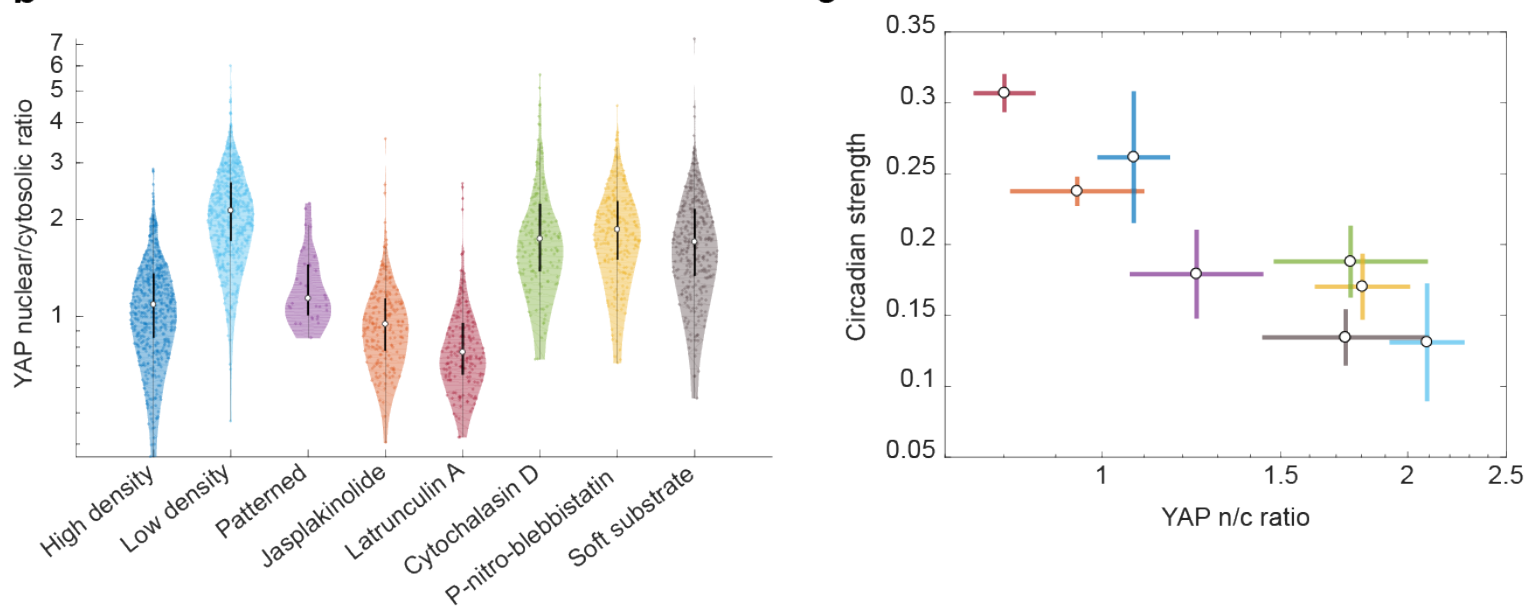

Fig. 3. Rev-erba circadian strength anticorrelates with nuclear YAP. a, Confocal microscopy images of cells under different conditions (high-density control, low-density control, micropatterned cells, low-density treated for 24 hours with jasplakinolide $1 \mu \mathrm{M}$, latrunculin A $200 \mathrm{nM}$, cytochalasin D $1 \mu \mathrm{M}$, para-nitro-blebbistatin $10 \mu \mathrm{M}$ and low-density cells grown on polyacrylamide gels with a stiffness of $300 \mathrm{~Pa}$ ). The cells were stained with an anti-YAP antibody (green, top) and Hoechst (blue, bottom; the cell perimeter is represented with a dashed white line. Scale bar, $20 \mu \mathrm{m}$. b, Violin plots representing the distribution of the single-cell YAP nuclear to cytosolic ratios for the conditions depicted in $\mathbf{a} ; \mathrm{n}=554,589,31$, 203, 171, 238, 153 and 315 cells, respectively, from 3 to 7 experiments depending on the condition. Medians and interquartile ranges are depicted as white circles and black bars, respectively. c, Correlation between circadian strength and YAP nuclear to cytosolic ratio of all the aforementioned conditions. The values represented are the means of the medians of each independent experiment for every condition. The error bars refer to the corresponding standard deviations. Pearson's correlation coefficient is $r=-0.89$ with a $p$-value of 0.002 . 


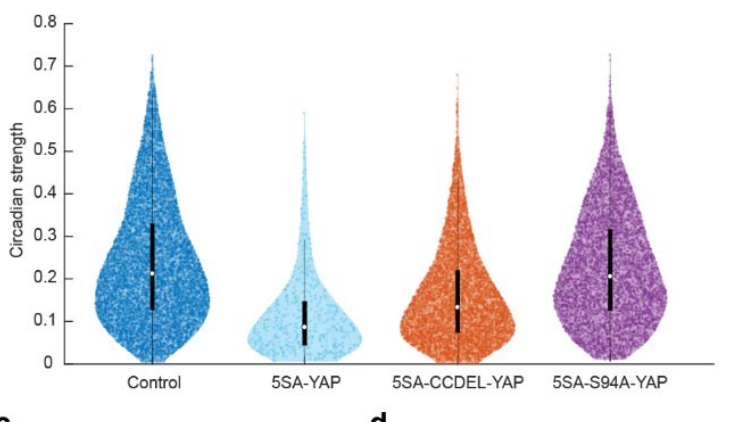

c

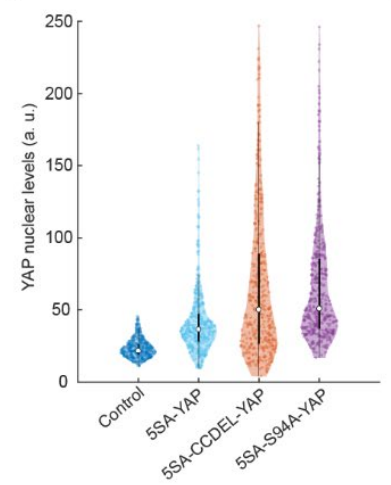

d

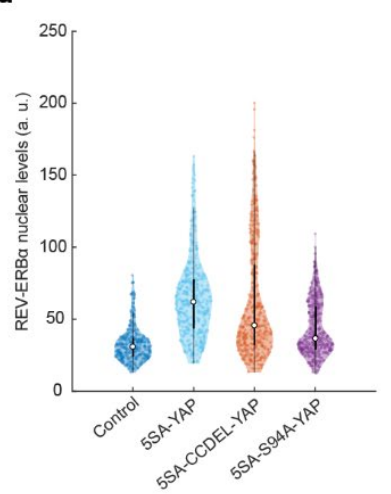

b
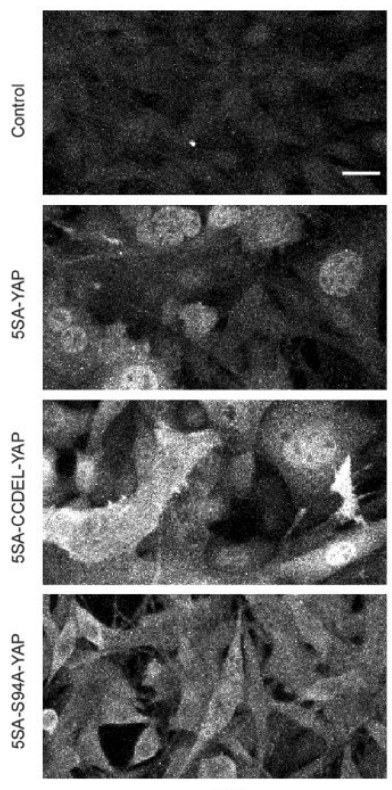

YAP
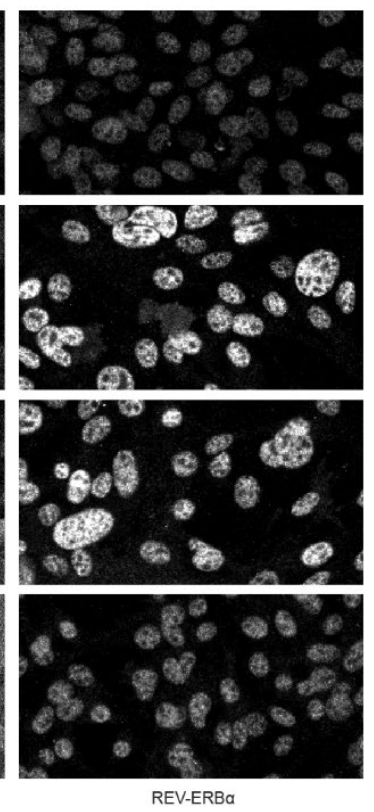

Fig. 4. YAP perturbs the circadian clock via TEAD. a, Violin plots representing the distribution of the single-cell RevVNP circadian strengths of YAP-overexpressing cells carrying different mutations and the control; $\mathrm{n}=6466,440,5320$ and 4571 for control, 5SAYAP, 5SA-CCDEL-YAP, and 5SA-S94A-YAP, respectively, from five independent experiments; Medians and interquartile ranges are depicted as white circles and black bars, respectively. Full p-values are reported in Extended Data Table 1. b, Confocal microscopy images of control, 5SA-YAP, 5SA-CCDEL-YAP, and 5SA-S94A-YAP cells immunostained for YAP (left) and a REV-ERB $\alpha$ (right). All images are displayed under the same brightness and contrast settings. Scale bar, $20 \mu \mathrm{m}$. c-d, Violin plots representing the single-cell levels of nuclear YAP (c) and nuclear REV-ERB $\alpha(\mathbf{d})$ for all the conditions depicted in $\mathbf{b} . \mathrm{n}=294,400$, 383 and 393 for control, 5SA-YAP, 5SA-CCDEL-YAP, and 5SA-S94A-YAP, respectively, from three independent experiments. Medians and interquartile ranges are depicted as white circles and black bars, respectively. Full p-values are reported in Extended Data Table 1. 


\section{Methods}

\section{Cell culture}

We cultured NIH3T3 mice fibroblasts in Dulbecco's Modified Eagle Medium (DMEM) with pyruvate (41966-029, ThermoFisher) supplemented with $10 \%$ or $2 \%$ FBS (for cell lines maintenance or for experimental purposes, respectively; 10270-106, ThermoFisher), $100 \mathrm{U} \mathrm{ml}^{-1}$ penicillin, $100 \mu \mathrm{g} \mathrm{ml}^{-1}$ streptomycin and $292 \mu \mathrm{g} \mathrm{ml}^{-1}$ of L-glutamine (10378016, ThermoFisher). For all the experiments except the gap closure one, thymidine $2 \mathrm{mM}$ (T9250$1 \mathrm{G}$, Sigma) was added 24 hours before fixation or image acquisition. The experiment with conditioned medium was carried out with medium composed by $50 \%$ collected medium from a high-density population of cells after two days of culture and $50 \%$ fresh medium.

\section{Cell lines and constructs}

For the circadian analyses we generated a stable cell line expressing venus fluorescent protein with a nuclear localization signal (NLS) and a destabilization PEST domain under the promoter of Rev-erb $\alpha$ via lipofectamine-based transfection (Lipofectamine 3000, ThermoFisher) of the plasmid RevVNP, courtesy of Prof. Ueli Schibler ${ }^{20}$. Lentiviral particles carrying plenti6-H2BmCherry (Addgene plasmid $\# 89766^{47}$ ) were generated by transient transfection of Hek293T cells together with the corresponding envelope and packaging vectors. Several clonal lines were obtained by transduction of the RevVNP cell line with the mentioned particles and flow cytometry sorting (Beckman Coulter). For reproducibility reasons, one of them was selected and used for all the experiments.

The 5SA-YAP, 5SA-CCDEL-YAP, 5SA-S94A-YAP, 4SA-TAZ and control cell lines were obtained by transduction with retroviral particles generated in Hek293T cells and sustained selection with puromycin $2 \mu \mathrm{g} \mathrm{ml}^{-1}$ (A1113803, ThermoFisher). Specifically, the retroviral FLAG-5SA-YAP and FLAG-5SA-S94A-YAP plasmids were generated via restriction-based cloning from pQCXIH-Myc-YAP-5SA (Addgene plasmid \#33093 ${ }^{34}$ and pCMV-Flag-YAP5SA/S94A (Addgene plasmid \#33103 ${ }^{40}$, respectively, and pBABE-PURO (Addgene plasmid $\# 1764^{48}$ ). The CCDEL deletion was introduced in the latter via overlapping PCR with the primers YAP-DEL-CC-HA-F and YAP-DEL-CC-HA-R as described in ${ }^{30}$. The FLAG-5SAS94A-YAP plasmid corresponds to the Addgene plasmid The FLAG-4SA-TAZ plasmid was a kind gift of Dr. Hyun Woo Park (Yonsei University, Seoul, Korea ${ }^{35}$ ). All the cell lines were checked by PCR, DNA sequencing and Western blot.

The cytoskeletal drugs used throughout the study were: jasplakinolide $1 \mu \mathrm{M}$ (J4580-100UG, Sigma), cytochalasin D $1 \mu \mathrm{M}$ (C8273-1MG, Sigma), latrunculin A $200 \mathrm{nM}$ (L5163-100UG, Sigma) and para-nitro-blebbistatin $10 \mu \mathrm{M}$ (DR-N-111, OptoPharma). The control conditions were performed with similar concentrations of DMSO (D8418-50ML, Sigma). All of them were added 24 hours before the initiation of the live imaging or fixation.

\section{Time-lapse imaging of circadian oscillations of NIH3T3 fibroblasts}

Time-lapse acquisitions were performed on a scanning confocal microscope (Nikon Ti Eclipse) equipped with thermal, $\mathrm{CO} 2$, and humidity regulation. 12-well or 6-well glass-bottomed plates were fixed on the stage and imaging was performed using a $10 \times$ objective $(\mathrm{NA}=0.30$, air $)$ with 
focusing maintained by the Perfect Focus System (Nikon). The software NIS Elements was used to image every 15 minutes during experiments lasting for 3 to 5 days. Multiple wells, and multiple positions within the different wells, were imaged by phase contrast and in laser scanning mode using a $488 \mathrm{~nm}$ and a $561 \mathrm{~nm}$ excitation. For the $488 \mathrm{~nm}$ acquisition two different sets of settings were used, to better capture the large variations in fluorescence signal seen in the RevVNPchannel: a low-intensity channel with low excitation power, and a high-intensity channel with 5 times higher excitation power. All confocal images were acquired by scanning $1024 \times 1024$ pixels on the objective's field of view, yielding a pixel-size of 1.28um. Pixel dwell times were adjusted independently in each experiment.

\section{Single-cell tracking}

Tiff stacks of time-lapse microscopy images were analysed using Fiji ${ }^{49}$. Fluorescent nuclei visible in the high-intensity channel were tracked in space and time using the FIJI plugin TrackMate $^{50}$. Within TrackMate, spots were identified using the LoG detector, after which the LAP tracker was used, with a maximum linking distance of 15 pixels and gap closing set to 2 frames. The resulting tracks were then filtered by length, keeping only those that lasted at least 60 hours. In the rare cases of cell-division, only one daughter cell was tracked. The tracking data was then used to additionally obtain tracks for the low-intensity channel. Finally, the position and intensity data for both channels were exported as .csv files.

\section{Frequency Analysis of datasets of single-cell circadian oscillations}

All analyses were performed with custom software created using MATLAB R2017a. To calculate a cell's circadian strength firstly any missing time-points in each single-cell timeintensity track were interpolated. After this, each track was denoised by a low-pass filter, its mean value was subtracted, and the track was divided by its standard deviation. Then the tracks were analysed individually to remove any dying cells: these were defined as intensity traces whose standard deviation in the final 24 hours is less than $5 \%$ of the standard deviation during the rest of the experiment. A fast Fourier transform (FFT) was then applied, and the power spectral density (PSD) of each track was generated using the periodogram. All the PSDs were averaged, and the ensemble circadian frequency $f_{c}$ was identified as the coordinate of the peak in the interval $[0.7,1.3]$ days $^{-1}$ in the average PSD. The single-cell PSDs were then analysed as follows: the integral of the PSD in the frequency interval $\mathrm{W}=\left[f_{c}-0.2, f_{c}+0.2\right]$ days $^{-1}$ was divided by the total power of that individual track, giving what we termed the circadian strength of a single-cell oscillation. Note that to set the width of $\mathrm{W}$ we used pooled experiments with cells at high-density, identified the ensemble circadian frequency $f_{c}$ and measured the full width at half maximum of the corresponding peak.

\section{Kymography}

The denoised and rescaled single-cell tracks were further analysed by pairwise crosscorrelation, and the lag values giving maximum cross-correlation were obtained. By virtue of the periodicity of the signals, these lags were restricted to the interval [-1,1] days. Tracks were ordered by decreasing circadian strength and the lags with respect to the median track were used to shift the other tracks and thus synchronize the dataset. These data were plotted as a kymograph of fluorescence intensity by time and track-number. 
For the gap closure experiments, the nuclei in time-lapse fluorescence images were segmented using a combination of TrackMate's spot detection algorithm and intensity thresholding. The kymographs were generated by averaging the intensity of the segmented nuclei in each frame along the direction parallel to the wound edge. These lines were then assembled into a kymograph representing fluorescence intensity as a function of time and distance from the centre of the wound.

\section{Polyacrylamide gels}

Polyacrylamide (PAA) gels with Young modulus E= $30 \mathrm{kPa}$ - which matches approximately the stiffness of the majority of abdominal organs and the skin ${ }^{51}$ - were produced according to a previously published protocol ${ }^{52}$. A PBS solution containing $12 \%$ acrylamide (161-0140, BioRad), $0.15 \%$ bis-acrylamide (161-0142, Bio-Rad); plus 0.05\% ammonium persulphate (A3678, Sigma) and $0.05 \%$ tetramethyl ethylenediamine (T9281-25ML, Sigma) was prepared and allowed to polymerize between a coverslip and a glass-bottomed dish (Mattek). Alternatively, $3 \%$ acrylamide and $0.03 \%$ bis-acrylamide were used to make 300 Pa gels. The PAA gel surface was then incubated with a solution of $2 \mathrm{mg} \mathrm{ml}^{-1}$ Sulpho-SANPAH (4822589, ThermoFisher) under ultraviolet light for $5 \mathrm{~min}$ (wavelength of $365 \mathrm{~nm}$ at $5 \mathrm{~cm}$ distance). After that, the excess Sulpho-SANPAH was removed by three consecutive 3 min washes with PBS. A solution of fibronectin $0.1 \mathrm{mg} \mathrm{ml}^{-1}$ (F0895, Sigma) was added on top of the gels and left overnight at $4^{\circ} \mathrm{C}$.

\section{Gap closure}

The gap closure experiments were performed following the protocol described in ${ }^{53}$. Magnetic PDMS stencils consisting in two hollow regions separated by a barrier of 600-900 $\mu \mathrm{m}$ were obtained using a customized 3D-printed mould. The cured, autoclaved, passivated, and dried magnetic PDMS stencils were then deposited on a fibronectin-coated PAA gel polymerized in a glass-bottomed dish. After this, the dish was placed on top of a custom-made magnetic holder to keep the gasket in place and avoid medium leakage. The hollow regions were then filled with 40,000 cells contained in $120 \mu 1$ of medium. After 1 hour, excess of cells was removed by 2 consecutive washes with medium. The attached cells were left overnight at $37^{\circ} \mathrm{C}$ before the removal of the magnetic PDMS gasket and the filling of the whole glass-bottomed dish with medium.

\section{Immunostainings}

Cells were fixed using paraformaldehyde $4 \%$ for $15 \mathrm{~min}$ and washed with PBS three times. Then, they were permeabilized with $0.1 \%$ Triton X-100 for 45 min, incubated with the primary antibodies for $90 \mathrm{~min}$ at room temperature, washed and incubated with the secondary antibodies for another $90 \mathrm{~min}$ at room temperature. After this step, Hoechst 33342 (H3570, ThermoFisher) was added at a concentration of $1 \mu \mathrm{g} \mathrm{ml}^{-1}$ for 10 minutes. Finally, cells were mounted in Mowiol reagent (81381, Merck). The buffers used during the whole procedure had fish gelatine $1.6 \%(\mathrm{v} / \mathrm{v})$ as a blocking agent (G7765, Merck). The primary antibodies used were anti-NR1D1 (ab174309, abcam), anti-MKL1 (ab49311, abcam) and anti-YAP 63.7 (sc101199, Santa Cruz). The secondary antibodies used were Alexa Fluor-488 anti-mouse (A11029, ThermoFisher), Alexa Fluor-488 anti-rabbit (A-21206, ThermoFisher), Alexa Fluor555 anti-mouse (A-21424, ThermoFisher) and Alexa Fluor-555 anti-rabbit (A-21429, 
ThermoFisher). All the antibodies were incubated in a 1:200 dilution. The acquisition of $z-$ stacks (with a z-axis step of $0.7 \mu \mathrm{l}$ ) was done using a 40x water immersion LD LCI Plan Apo objective $(\mathrm{NA}=1.2)$ in a scanning confocal microscope with Fast AiryScan (Zeiss LSM880) or a $60 \mathrm{x}$ oil immersion objective $(\mathrm{NA}=1.40)$ in an inverted microscope (Nikon Eclipse Ti). Maximal projections of the stacks were obtained with Fiji before quantification of nuclear and cytosolic intensity levels or, for the case of YAP and MAL nuclear to cytosolic ratios, they calculated after measuring the mean intensity of two adjacent regions of identical size, inside and outside the nucleus as in ${ }^{54}$ using the Hoechst image as a reference for the nuclear position.

\section{Micropatterning}

The micropatterning protocol was an adaptation of ${ }^{55}$. Single glass-bottomed dishes $(35 \mathrm{~mm}$ Dish, No. 0 Coverslip, $10 \mathrm{~mm}$ Glass Diameter, MatTek) were cleaned with ethanol 96\%, dried, and oxidized by exposure to oxygen-plasma (PCD-002-CE, Harrick) for $30 \mathrm{~s}$ at $7.2 \mathrm{~W}$. After that, a ring of PDMS with an inner diameter of $7 \mathrm{~mm}$ was attached to the coverslip to delimitate the micropatterning area, and that area was immediately filled with $30 \mu 1$ of PLL-PEG solution (PLL (20 Kda)-g[3,5]-PEG(2), SuSoS) at a concentration of $0.1 \mathrm{mg} \mathrm{ml}^{-1}$ in PBS, which was incubated for $1 \mathrm{~h}$ at room temperature. Then, the solution was carefully removed, the surface was washed three times with PBS and $30 \mu \mathrm{l}$ of photoinitiator PLPP (4-benzoylbenzyltrimethylammonium chloride; Alvéole) at a concentration of $14.5 \mathrm{mg} \mathrm{ml}^{-1}$ were added. After that, micropatterning was performed in an inverted microscope (Nikon Ti Eclipse), using the PRIMO system controlled by the Leonardo software (Alvéole) and a 20X objective (CFI S Plan Fluor ELWD ADM, Nikon), directing a UV power of $1050 \mathrm{~mJ} \mathrm{~mm}$ following the patterns predesigned in ImageJ. When the patterning was completed, filtered PBS (concentration 1X) was used to clean three times and the PDMS ring was removed. The whole glass surface was then treated for 10 min with a solution of fibronectin (F0895, Sigma-Aldrich) $100 \mu \mathrm{g} \mathrm{ml}^{-1}$ and Alexa Fluor 647 fibrinogen (F35200, ThermoFisher) $5 \mu \mathrm{g} \mathrm{ml}^{-1}$, which caused attachment of the coating proteins at both the patterns and the area where the PDMS ring had previously been placed. After three washes, the glass-bottomed dishes were stored, filled with PBS, at $4{ }^{\circ} \mathrm{C}$ for a maximum of 48 hours before the experiments. $2 * 10^{5}$ cells were seeded diluted in DMEM with $0.2 \%$ FBS to avoid cell attachment in unwanted areas. After one hour, excess of cells was removed by three intensive washes with the same medium. 24 hours before imaging or fixation the medium was replaced with $2 \%$ FBS-containing DMEM.

\section{Statistical analysis}

All violin plots were generated using violinplot, an open-source MATLAB script ${ }^{56}$. Two-sided Wilcoxon rank sum test were used when testing for statistical significance, and $p$-values smaller than 0.05 were considered as significant, unless specified otherwise. 


\section{Methods references}

47. Pemble, H., Kumar, P., van Haren, J. \& Wittmann, T. GSK3-mediated CLASP2 phosphorylation modulates kinetochore dynamics. J. Cell Sci. (2017) doi:10.1242/jcs.194662.

48. Morgenstern, J. P. \& Land, H. Advanced mammalian gene transfer: High titre retroviral vectors with multiple drug selection markers and a complementary helperfree packaging cell line. Nucleic Acids Res. 18, (1990).

49. Schindelin, J. et al. Fiji: An open-source platform for biological-image analysis. Nature Methods (2012) doi:10.1038/nmeth.2019.

50. Tinevez, J. Y. et al. TrackMate: An open and extensible platform for single-particle tracking. Methods 115, (2017).

51. Guimarães, C. F., Gasperini, L., Marques, A. P. \& Reis, R. L. The stiffness of living tissues and its implications for tissue engineering. Nature Reviews Materials vol. 5 (2020).

52. Bazellières, E. et al. Control of cell-cell forces and collective cell dynamics by the intercellular adhesome. Nat. Cell Biol. 17, 409-20 (2015).

53. Rodriguez-Franco, P. et al. Long-lived force patterns and deformation waves at repulsive epithelial boundaries. Nat. Mater. (2017) doi:10.1038/NMAT4972.

54. Elosegui-Artola, A. et al. Force Triggers YAP Nuclear Entry by Regulating Transport across Nuclear Pores. Cell 171, 1397-1410.e14 (2017).

55. Strale, P. O. et al. Multiprotein Printing by Light-Induced Molecular Adsorption. Adv. Mater. (2016) doi:10.1002/adma.201504154.

56. Bechtold, B. Violin plots for MATLAB. Github Proj. (2016).

\section{Acknowledgments}

We thank S. Aznar-Benitah, G. Solanas, M. Uroz, A. Elósegui-Artola, C. Pérez-González, D. Zalvidea and all the members of the Roca-Cusachs and Trepat laboratories for their discussions and support. We thank A. Menéndez, S. Usieto and N. Castro for daily technical assistance. We also thank N. Montserrat, E. Martínez, U. Schibler, H.W. Park and Y.S. Choi for sharing some of the cell lines and plasmids used in this work. This paper was funded by The Generalitat de Catalunya (AGAUR SGR-2017-01602 to X.T, AGAUR Beatriu de Pinós 2014 BP-B 00105 to J.F.A., the CERCA Programme, and "ICREA Academia" awards to P.R-C. and J.G.-O.); Spanish Ministry for Science and Innovation MICCINN/FEDER (PGC2018-099645-B-I00 to X.T., BFU2016-79916-P and PID2019-110298GB-I00 to P. R.-C, and PGC2018-101251-BI00 to J.G.-O.); European Research Council (Adv-883739 to X.T.); Fundació la Marató de TV3 (project 201903-30-31-32 to X.T.); European Commission (H2020-FETPROACT-01-2016731957 to P.R-C. and X.T., H2020 Marie Sklodowska-Curie Actions MECHADIAN - 
IF/750557 to J.F.A.); La Caixa Foundation (LCF/PR/HR20/52400004 to to P.R-C. and X.T.); IBEC is recipient of a Severo Ochoa Award of Excellence from the MINECO. DCEXS-UPF is recipient of a Maria de Maeztu Award of Excellence from the MINECO.

\section{Author contributions}

J.F.A. and X.T. conceived the project. J.F.A., M.M., L.R. and I.A. performed experiments and analysed data. L.R. and J.B. developed software and analysed data. K.K., P.R.-C., S.M., and J.G.-O. contributed technical expertise, materials, and discussion. J.F.A., L.R. and X.T. wrote the manuscript. J.F.A. and X.T. supervised the project. All authors revised the completed manuscript.

\section{Competing interests}

The authors declare no competing financial interests 